\title{
The case of Schnitzler syndrome in one single rheumatologic center
}

\author{
S Salugina ${ }^{1 *}$, E Fedorov$^{1}$, V Gorodetskiy ${ }^{2}$, M Evsikova $^{2}$, N Lopatina ${ }^{2}$ \\ From 8th International Congress of Familial Mediterranean Fever and Systemic Autoinflammatory Diseases \\ Dresden, Germany. 30 September - 3 October 2015
}

\section{Background}

Schnitzler syndrome is characterized by chronic, nonpruritic urticaria in association with recurrent fever, bone pain, arthralgia or arthritis, and a monoclonal immunoglobulin M (IgM) gammopathy. Pathogenesis of Schnitzler syndrome is unclear. Some hypothesize that the deposition of the IgM paraprotein, leading to the formation of immune complexes and the activation of the complement cascade, is responsible for the cutaneous manifestations. Another proposed mechanism involves the uncontrolled activation of interleukin 1-alpha (IL-1 $\alpha$ ).

\section{Aim}

To report the case of Schnitzler syndrome in our clinic, that to be considered like rare entities.

\section{Case report}

A 44-year-old Caucasian man had had symptoms beginning at the age of 40 years, including fever, fatigue, recurrent urticaria, conjunctivitis, swelling of the eyelids, angioedema sometimes. Urticaria lasted 2-3 days and then disappeared without sequelae. These episodes recurred once per month. The laboratory findings included an ESR of up to $36 \mathrm{~mm} / \mathrm{h}$, neutrophil leukocytosis - 12.4-18.7 × 109, C-reactive proteine rise $-107 \mathrm{mg} / \mathrm{l}$ (normal 0-5.0 mg/l), ferritin - $415 \mathrm{mkg} / \mathrm{l}$ (normal 20-150 $\mathrm{mkg} / \mathrm{l})$, , SAA-127 ng/ml (normal 0-6, $4 \mathrm{ng} / \mathrm{ml}$ ), ANA and RF were negative, a monoclonal immunoglobulin $M$ (IgM) gammopathy detected with serum immunoelectrophoresis in a concentration $5 \mathrm{~g} / \mathrm{L}$. A histopathologic examination was not done. The patient was treated with nonsteroidal anti-inflammatory drugs (NSAIDs), systemic steroids intravenously (120-90-60 mg) and orally (20 mg), methotrexate $20 \mathrm{mg} /$ week, that were somewhat effective at controlling the urticaria and fever. Differential diagnoses was made with Systemic Lupus Erythematosus, Still's disease in adult, Acute Urticarial Vasculitis, also hereditary autoinflammatory diseases - cryopyrinassociated periodic syndromes (CAPS) - Muckle-Wells syndrome (MWS).

A genetic analysis on the patient did not show a mutation in the NLRP3 (CIAS1), MVK, TNFRSF1A genes.

\section{Conclusions}

We report the rare case of Schnitzler syndrome. It was very difficult to diagnose and confirm this diagnosis. The clinical and laboratory signs are wery similar with MWS. But later then in pts with MWS adult age (40 years old) of onset of disease and also a monoclonal immunoglobulin M (IgM) gammopathy put the correct diagnosis.

\section{Consent to publish}

Written informated consent for publication of their clinical details was obtained from the patient/parent/guardian/ relative of the patient.

\section{Authors' details}

${ }^{1}$ Nasonova Research Institute of Rheumatology, Pediatric Department, Moscow, Russian Federation. ${ }^{2}$ Nasonova Research Institute of Rheumatology, Rheumatology Department, Moscow, Russia, Moscow, Russian Federation.

Published: 28 September 2015

doi:10.1186/1546-0096-13-S1-P164

Cite this article as: Salugina et al:: The case of Schnitzler syndrome in one single rheumatologic center. Pediatric Rheumatology 2015

13(Suppl 1):P164. 